\title{
Regionalization for urban air mobility application in metropolitan areas: case studies in San Francisco and New York
}

\author{
Namwoo Kim, Yoonjin Yoon \\ Department of Civil and Environmental Engineering, Korea Advanced Institute of Science and Technology (KAIST), Daejeon 34141 Korea
}

\begin{abstract}
In a new era of mobility where the transportation of persons or goods via flying vehicles over urban areas has garnered great interest in its application in urban space. With the anticipated utilization of sUAS in urban airspace, a multi-dimensional understanding of urban space is essential. As a first step to assess the feasibility of Urban Air Mobility (UAM) in urban areas, we conduct regionalization and correspondence analysis in highly urbanized areas - San Francisco, CA and Manhattan, NY - by incorporating population dataset and urban 3D airspace to delineate the regional boundaries. Regionalization is carried out using graph-based clustering technique called SKATER (Spatial 'K'luster Analysis by Tree Edge Removal) to group the regions having similar characteristics and address the compound effect of both population and spatial information. By classifying the regions into five categories through correspondence analysis, the operational and economic feasibility of each region is evaluated. The results provide the region maps of each city with the most and least attractive regions for UAM application with the temporal notion, whether the clusters are daytime-intensive or nighttime-intensive areas. The outcomes have several unique information that can benefit drone delivery target area identification, landing location identification, demand prediction. Our approach can contribute to providing a useful basis for management for UAM in urban areas as well as the process of regulating airspace use.
\end{abstract}

Keywords: Urban Air Mobility; UAS Traffic Management; Geodemographic Analysis; Spatial Clustering

\section{Introduction}

The conventional usage of the term urban space in research domain has been limited to land transportation, economic development and urban design [1, 2]. The road congestion in the existing two-dimensional urban space and advances in aviation technologies contribute to bringing urban mobility into the third dimension [3, 4]. With the new entrants — Urban Air Mobility (UAM) - to urban airspace, the need to expand the research beyond the surface transportation environment is evident [5]. In urban airspace filled with manmade structures and terrain, several researches are actively underway on the three most difficult UAM use cases, including last mile delivery, air subway and air taxi [6].

With the spatial extent that UAM can safely navigate, there have been several notable research efforts of attempts to assess the operational feasibility of UAM applications recently. Cho and Yoon defined two categories of urban airspace - free and usable, where free airspace is the airspace with no physical interruption, and equivalent to the empty space as human eyes recognize [7, 8]. Usable airspace is the space that UAM can fly in. For instance, under the airspace restriction, usable airspace is the space after removing the geofenced spaces out of the free airspace. Distinction between the free and usable airspace is particularly relevant in the urban environment, where high-rise buildings are often closely located in the prime real-estate areas. A similar approach to assess usable airspace was conducted for San Francisco Bay Area airspace up to 5,000 ft considering airspace restrictions such as airport controlled airspace, special use airspace, and flight procedures [9]. However, those studies have focused on methodology to extract usable airspace in the environment of complex geospatial characteristics with limited applications to the real urban environment.

While the operational feasibility of UAM is well addressed by airspace restriction in many studies, it is important to incorporate demand perspective in enabling viable UAM applications. In other words, for reasonable assessment of both the operational and economic feasibility, population information needs to be integrated in association with the usable airspace. From [6], demand was used as one of the important components of econometric models to assess UAM market feasibility in 5 representative cities including New York City and San Francisco. In our research, we used population dataset as a way to represent potential demand, and temporal variations of the population during daytime and nighttime were incorporated.

\footnotetext{
* Corresponding author. Tel.: +82 423503655 ;

Fax: +82 42350 3610. E-mail address: yoonjin@kaist.ac.kr

(C) 2021 International Association for Sharing Knowledge and Sustainability.

DOI: 10.5383/JTTM.03.02.001
} 


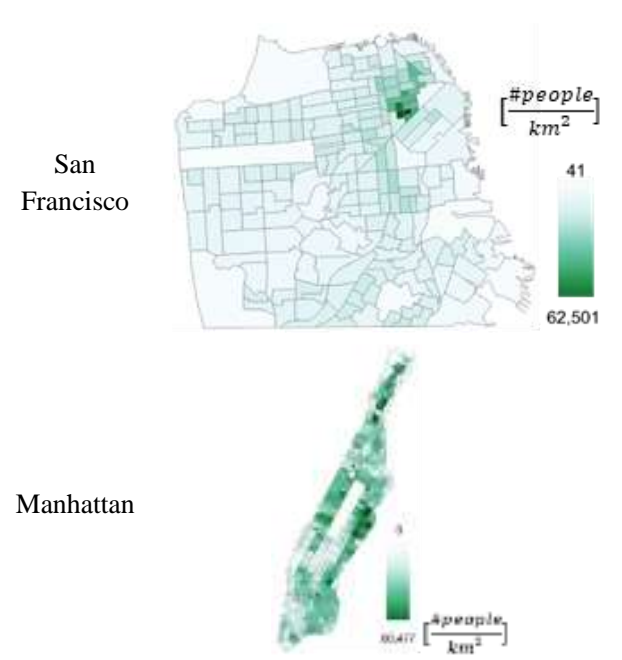

(a) $\rho_{N}$

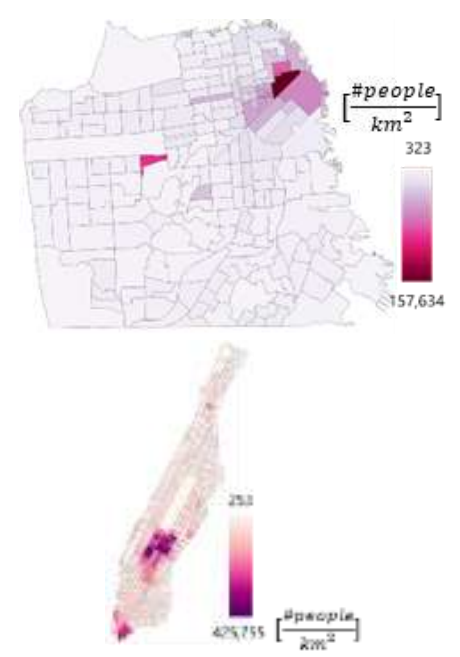

(b) $\rho_{D}$

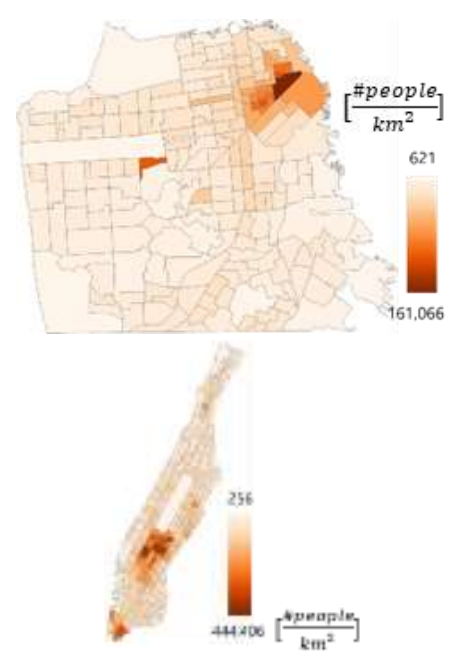

(c) $\rho$

Fig. 1 Choropleth maps for (a) Error! Reference source not found., (b)Error! Reference source not found., and

As a first step to assess the feasibility of UAM in urban areas, we conduct 3D geodemographic analyses of two major cities in San Francisco, CA and Manhattan, NY. The 3D building footprint data is used to identify the raw available airspace as well as the added spatial restrictions with geofencing. In addition, we proposed to group the areas of similar spatial and population characteristics through regionalization. Regionalization $[10,11]$ is a spatially constrained multi-variate clustering method to group small geographical units (census blocks and tracts in general) into a contiguous region of homogeneous nature. The main benefit of regionalization is to delineate regions of similar characteristics and spatial proximity $[12,13]$. Through regionalization, one can better understand the urban space with comprehensive geographic perspective, rather than a small geographical unit of census blocks or tracts. We adopted the SKATER [14], an efficient regionalization technique that uses minimum spanning tree consisting of a connected tree with no circuits. The intention is to provide a region map of the city that can readily identify regions of similar UAM operational and population characteristics with spatial continuity and feasibility. Based on the regionalization results, correspondence analysis was conducted to translate the compound effect of spatial and population characteristics into feasibility.

This paper is organized as follows. In section 2 and 3, we provide the data description and the summary on the SKATER method, respectively. Analysis of San Francisco and Manhattan is presented in section 4. In section 5, operational and economic feasibility analysis is presented and compared between two cities using correspondence analysis. In the final section, conclusions and discussions are presented.

\section{Data Description}

One of the main objectives of this study is to utilize a diverse set of data to model the interaction of urban population and the 3D airspace. The main source of the 3D geospatial characteristics is the building dataset, and two types of population data - the daytime and nighttime population, were incorporated to address temporal variations of urban population. By considering both the daytime and nighttime population with the $3 \mathrm{D}$ geospatial information, we aim to evaluate the temporal variations of such interactions in populated urban regions.

\subsection{Population Data}

The population data was sourced from 2016 Esri demographic datasets [15], which contains both the daytime and nighttime population per census tract. Daytime population covers the total number of residents and workers present during normal business hours. Nighttime population covers residential population. The daytime and nighttime population are estimated value from the decennial census, American Community Survey (ACS), and business data from Infogroup. In Fig. 1, the population density (Error! Reference source not found.) of the nighttime Error! Reference source not found., daytime Error! Reference source not found., and total population Error! Reference source not found. of each city is shown by census tracts.

\subsection{Geospatial Information}

The 3D geospatial characteristics of San Francisco and Manhattan are sourced from the open data portals [16, 17], which contain the footprint and height information of 3D building structures. In Fig. 2, the building height distributions of San Francisco, CA and Manhattan, NY are presented. Although one can identify the obstacle-free airspace simply by taking the volume of 3D structures off the airspace boundary, there is another factor to consider for UAM applications in addition to the physical obstacles. It is conventional to impose minimum safety margin of virtual buffer around the obstacles, or geofence, for safety and privacy purpose. In regard to the two cities included in the study, such safety margin is not specified by the U.S. government. However, several countries require the minimum of 30 meters geofence volume for vehicles to stay out of, and the same standard is considered in our analysis $[18,19]$. 


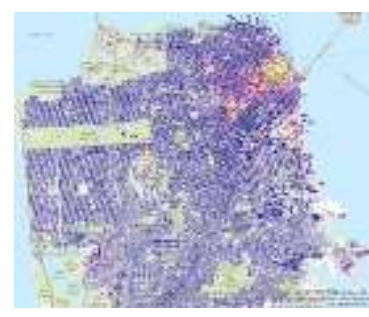

(a): San Francisco, CA

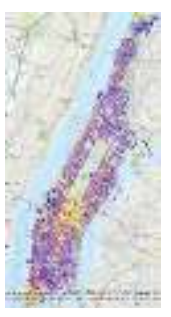

(b): Manhattan, NY
Fig. 2 Building height distribution of San Francisco and Manhattan

In Fig. 3, the effect of geofence is projected in 2D, where static obstacles are shown in black and the geofenced area is shown in grey. Note the effect of geofence reduces the available area, and therefore increases restriction, as shown in Fig. 3 (b). In this study, the airspace restriction is evaluated in both categories of the raw obstacle-free airspace Error! Reference source not found. and the airspace with geofence of 30 meters (Error! Reference source not found.). Computing the 3D geofence around the predefined geometry was carried out by Minkowski sum of a disk and a geometry.

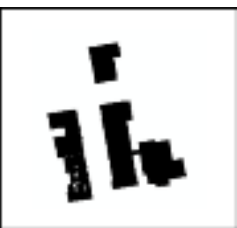

(a): without geofence

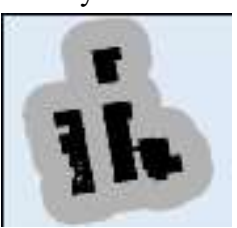

(b): with geofence
Fig. 32 Drojection of the effect of geofence.

Note that the spatial dataset is vectorized, whereas the population data is available in census tract. Since the resolution of two data sources differ, spatial information was aggregated in census tract. To normalize variances, airspace availability was measured as available percentage

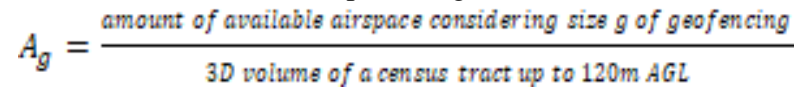
, and population is measured as population density per census tract Error! Reference source not found.. In Fig. 4, airspace availability choropleth maps of each city are presented.
Among the 176,366 building structures included in San Francisco, the majority of tall buildings are located in the Financial District. Once excluding those regions, the majority of San Francisco is characterized by loosely distributed lowrise buildings with green space. The citywide raw obstacle-free airspace Error! Reference source not found. is $96.71 \%$, which essentially captures the amount of open airspace to human perception up to 400 feet. With geofence application of $30 \mathrm{~m}$, the availability reduces about $7.2 \%$ to $89.71 \%$. In the city, most census tracts show more than $90 \%$ airspace availability except for several tracts near Financial District. Once the geofence of 30m is applied, reduction from Error! Reference source not found. to Error! Reference source not found. ranges from 0.7 to 40.25 percentage point. The tract with the largest reduction is located in the Financial District, where Error! Reference source not found. $=70.69 \%$ and Error! Reference source not found. $=30.44 \%$. Also note that the effect of geofence is negligible in the most areas of the city with average reduction is $7.57 \%$.

In case of Manhattan, a total of 45,712 buildings were extracted from the geodatabase. The raw obstacle-free airspace of Manhattan is $89.36 \%$ but reduced to $66.76 \%$ with the 30 meters geofence. The airspace reduction due to geofence is not proportional across the city. Once geofence is applied, reduction amount of each census tract from Error! Reference source not found. to Error! Reference source not found. is ranging from $1.27 \%$ to $59.54 \%$. Especially, census tracts located in the city centre are severely affected by geofence. The census tract with the largest reduction in airspace availability is in the Midtown, with Error! Reference source not found. $=68.94 \%$ and Error! Reference source not found. $=9.4 \%$. On the other hand, the airspace availability of northern Manhattan including central park is more than $70 \%$, regardless of geofence application. The effect of geofence in Manhattan, where the average reduction is $25.31 \%$, is observed to have a greater significance than in San Francisco.

\section{Regionalization}

Regionalization was carried out using SKATER, proposed by Assunção et al. in 2006. SKATER is a graph-based clustering

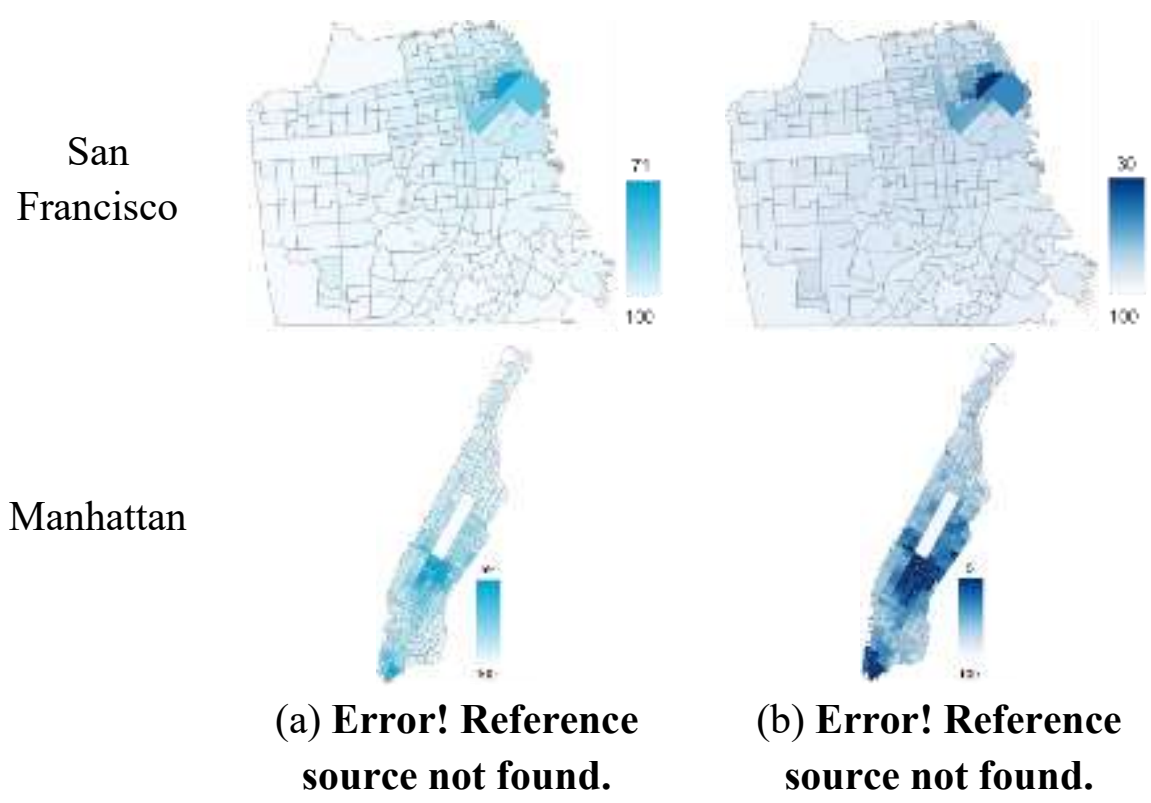

Fig. 4. Choropleth maps for (a) Error! Reference source not found. and (b) Error! Reference source not found. in San 


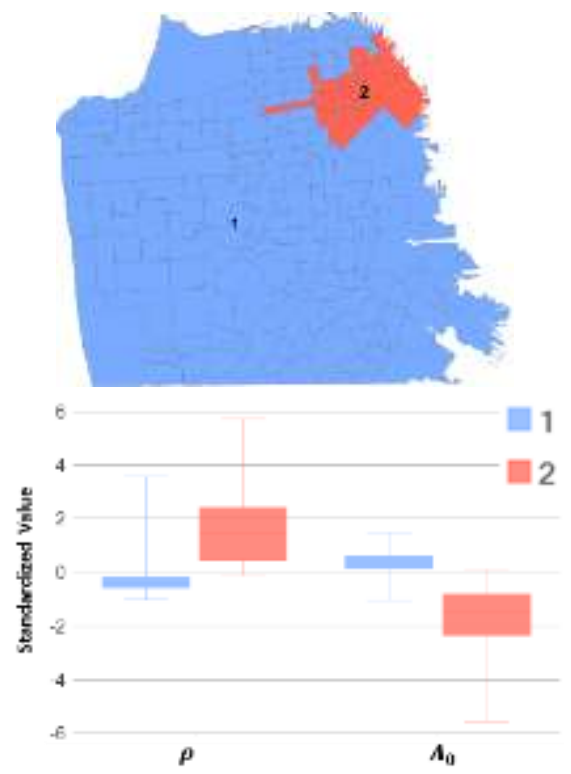

(a)

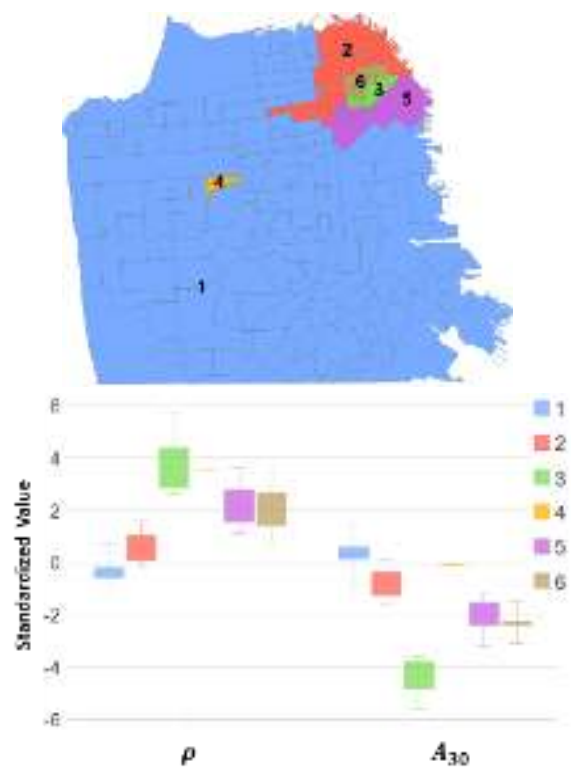

(b)

Fig. 5. Regionalization result and boxplot of San Francisco for (a) Error! Reference source not found. and (b) Error! Reference source not found..

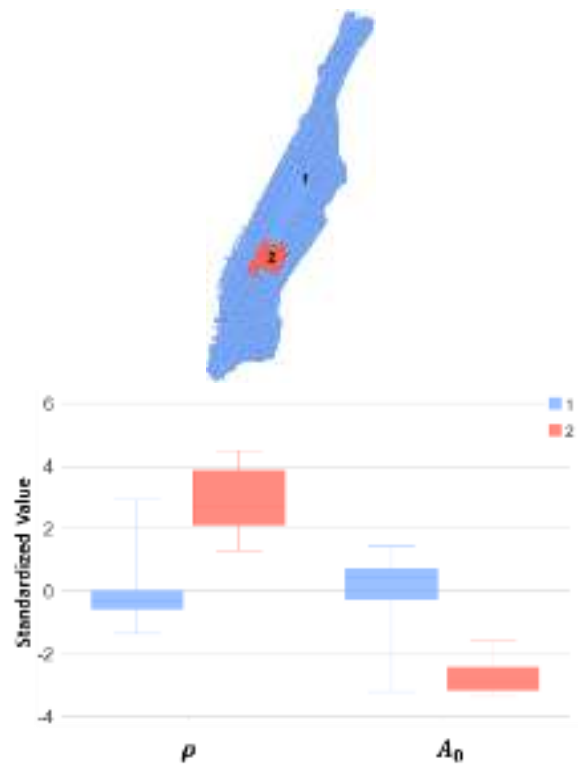

(a)

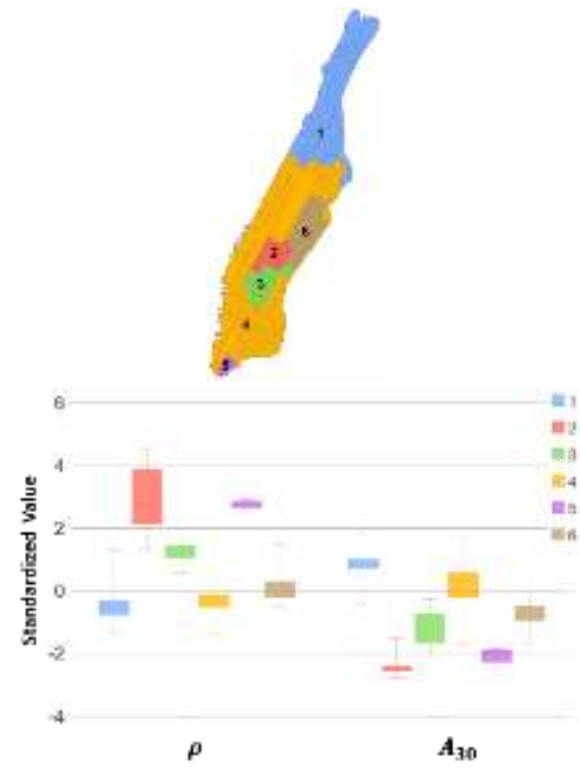

(b)

Fig. 6. Regionalization result and boxplot of Manhattan for (a) Error! Reference source not found. and (b) Error! Reference source not found..

technique that incorporates the geospatial contiguity using graph structure to generate clusters of regions of similar characteristics. SKATER first creates undirected weighted graph Error! Reference source not found. based on the node adjacency, where Error! Reference source not found. is the set of nodes and Error! Reference source not found. is the set of edges. Given Error! Reference source not found., edge weight Error! Reference source not found. is defined with the dissimilarity of two connected nodes. Minimum Spanning Tree (MST) is then obtained by pruning edges to minimize the sum of dissimilarities over all nodes through Prim's algorithm
[21]. Finally, the MST is partitioned into the set of sub-trees (cluster) that minimizes within-cluster sum of squares. In our analysis, the Error! Reference source not found. is the set of census tracts and Error! Reference source not found. is established based on the rook neighbors. The populations density Error! Reference source not found. and airspace availablility Error! Reference source not found. and Error! Reference source not found. are used as weights to define the weight function Error! Reference source not found.. In our research, the number of clusters (regions) was chosen by using jump index technique [21]. 


\section{Results}

To define the geospatial boundaries of similar characteristics both in airspace and population, SKATER is applied to the two combinations of airspace availability and total population Error! Reference source not found. and the not found. 1, 4, and 6). Even though cluster Error! Reference source not found. 2 and 5 have similar characteristics, they are not grouped as the same cluster due to the spatial disjointedness.

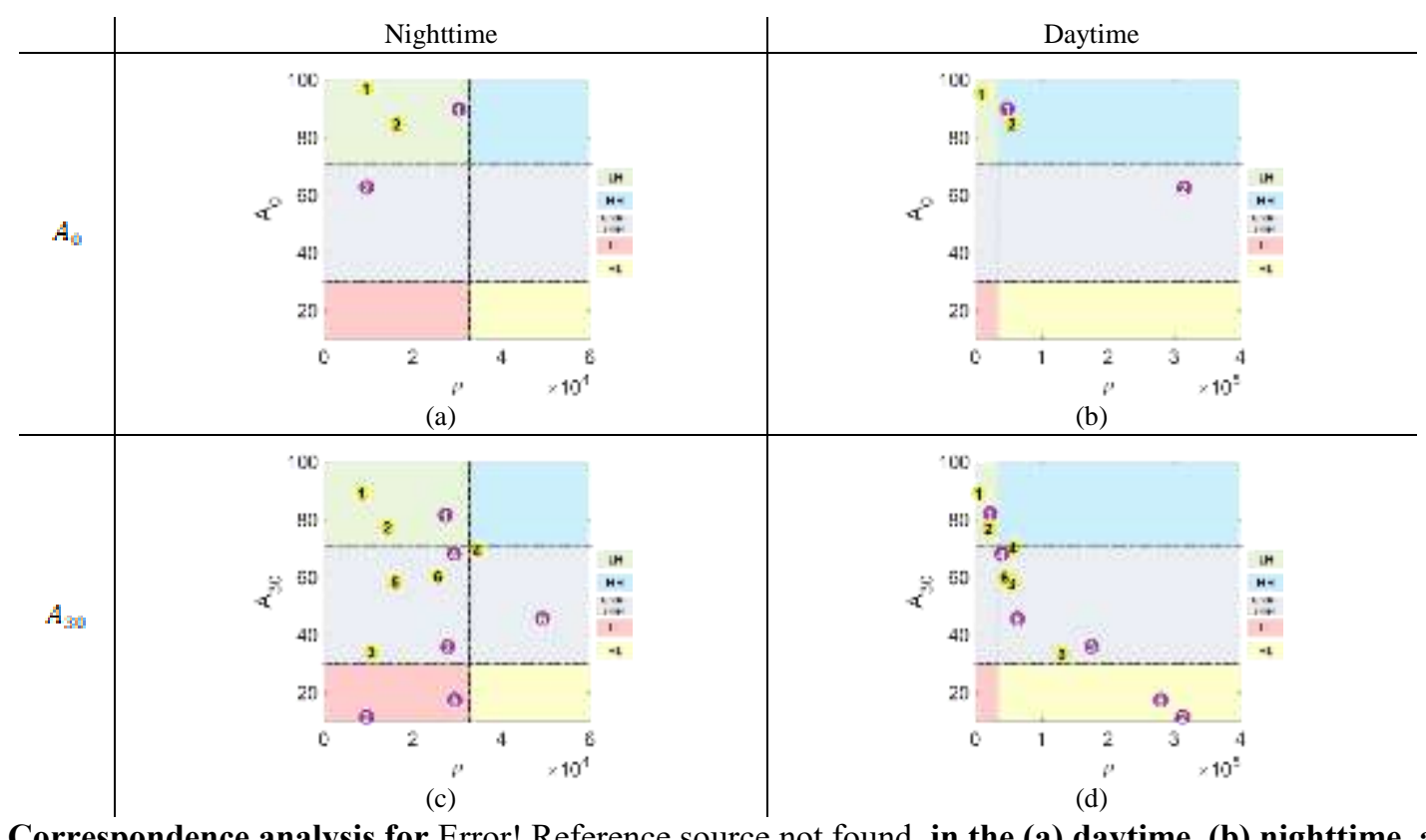

Fig. 7. Correspondence analysis for Error! Reference source not found. in the (a) daytime, (b) nighttime, and for

results are presented in Fig. 5 and 6.

In San Francisco, the entire city shows rather homogeneous characteristics except for the areas near the Financial Districts and Embarcadero (cluster Error! Reference source not found. 2). When geofence is applied, however, various regions are observed mainly in the northeast corner of San Francisco which includes the North, the Embarcadero and Financial District. We have two groups of regions; the high population group (clusters Error! Reference source not found. 2 to 6 ) and the low population group (cluster 1). In addition, the effect of geofence expanded the regional boundary of cluster 2 identified for non-geofenced airspace to include the Marina District and identified the Inner Sunset business district as a single tract cluster (cluster Error! Reference source not found.4).

Except for Midtown (cluster Error! Reference source not found. 2), the regionalization results show that Manhattan has rather homogeneous characteristics in terms of population and airspace availability as shown in Fig. 6. However, after applying geofence, regional boundaries change considerably. The effect of geofence subdivided cluster Error! Reference source not found. 1 into Northern Manhattan (cluster Error! Reference source not found. 1), Upper West Side (cluster Error! Reference source not found. 6), and Lower Manhattan- Chelsea - Hell's Kitchen -Upper West Side (cluster Error! Reference source not found. 4). Through regionalization, Manhattan was divided into a group with high population and low airspace availability (cluster Error! Reference source not found. 2, 3, and 5), and another group with opposite characteristics (cluster Error! Reference source

\section{Feasibility Analysis}

In this section, we apply the correspondence analysis to translate the compound effect of airspace restriction and population into feasibility. The idea is to classify the large range of continuous values resulting from airspaceError! Reference source not found.population analyses, as presented in the previous sections, into five simple categories of $\mathrm{HH}$ (High population/high airspace availability), LL (low population/low airspace availability), HL (High population/low airspace availability), LH (low population/high airspace availability) and the Undecided. HH category is the most ideal case with high airspace availability with large potential customer base. In the similar sense, LL category is the least desirable case with limited airspace and customer base. HL and LH present unbalanced cases between airspace restriction and population. In LH case, the airspace is mostly usable, but there is limited customer base. Such regions might require further exploration for suitable mission choice, such as infrastructure inspection or search-and-rescue operations. HL can be characterized as the elevated risk yet attractive area with large potential customer base with rather restricted airspace. Such environment is not too difficult to imagine in highly urbanized areas, especially around the busiest part of the city. In our analysis, two airspace restrictions of with (Error! Reference source not found.) and without (Error! Reference source not found.) geofence are considered in combination with the population density. By incorporating the geofence, one can evaluate how the area-specific feasibility changes once the 
safety restriction is put in place. In addition, we labeled the most attractive clusters with temporal notion, whether the clusters are daytime-intensive or nighttime-intensive areas. Such information can be used to infer the various applications
District, since it has high airspace availability and large potential demand. Unlike San Francisco, New York's main central business area, Midtown (NY: cluster2), was identified as Undecided. The operational feasibility of airspace restriction

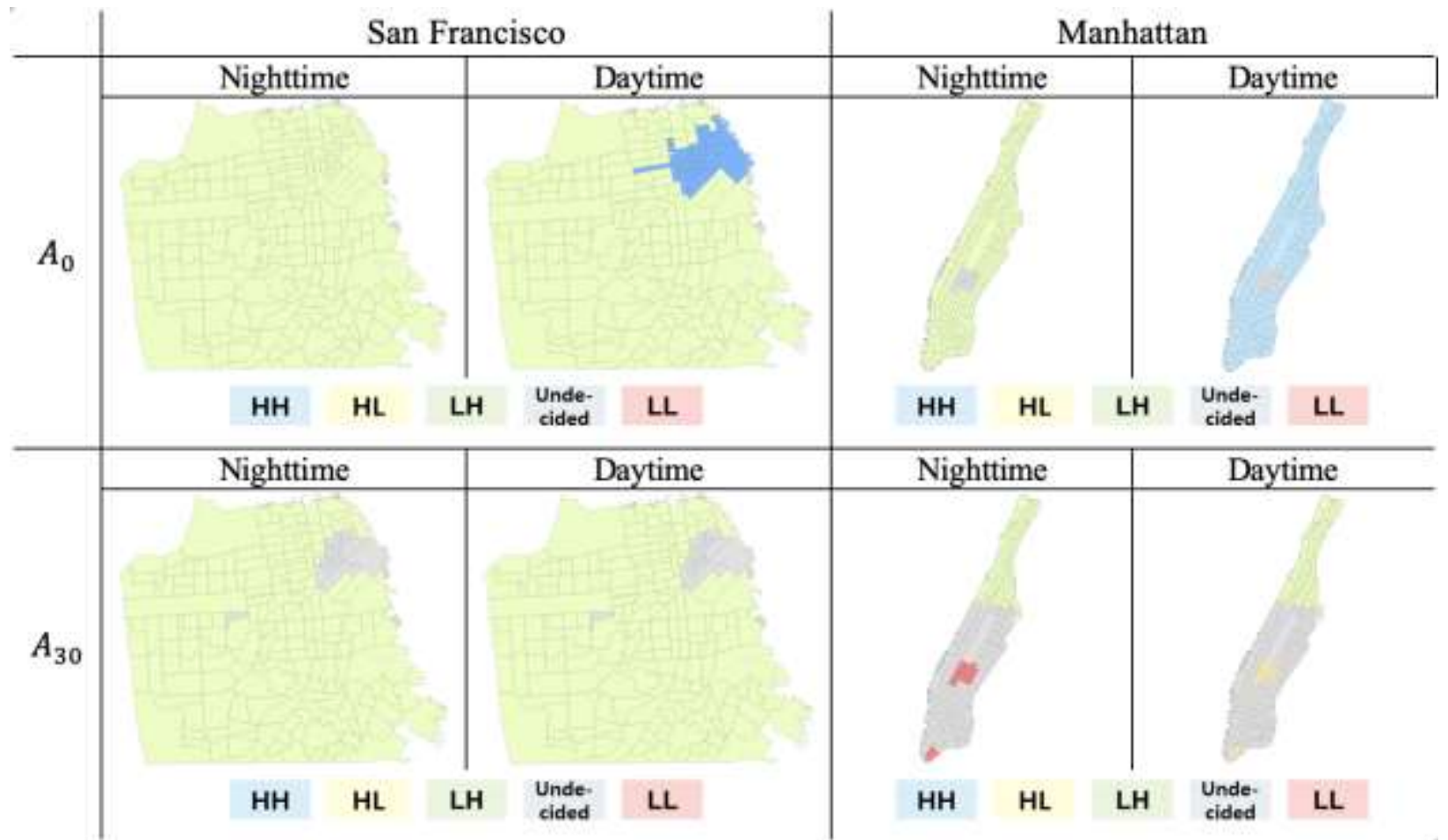

Fig. 8 Maps for five categories of San Francisco and Manhattan.

of target customers such as last-mile delivery point.

In Fig. 7, correspondence maps between airspace availability and population density are presented. In the plot, the city-wise clusters in Fig. 5 and 6 are plotted, and the five feasibility categories are color-coded. The threshold values to define the high and low airspace availability are chosen at $70 \%$ and $30 \%$, respectively for both cities. Threshold values for high population was set to 32,888 persons/Error! Reference source not found. according to the three urban population density categories of low (1,564/Error! Reference source not found.), medium (6,629/Error! Reference source not found.), and high (32,880/Error! Reference source not found.) presented in [22]. The aggregated values over each cluster for Error! Reference source not found. andError! Reference source not found. are represented in the airspace availability (Error! Reference source not found. or Error! Reference source not found.) Error! Reference source not found. daytime and nighttime population density (Error! Reference source not found. or Error! Reference source not found.) plane. The aggregated values over clusters of San Francisco and New York are marked in yellow and purple, respectively, with the corresponding cluster numbers. In addition, maps for five categories for each city are illustrated in Fig. 8.

In the daytime (Fig. 7 (b)), we observe only one cluster in each city that falls into the most attractive category, HH. SF's Financial District area (SF: cluster 2) is an active business area with a large population concentrated during the day. Without geofence, one can expect highest-value service in Financial becomes the main limiting factor in UAM application in active business area of Manhattan.

After application of geofence, since Manhattan and San Francisco are highly urbanized areas with many skyscrapers, airspace in those cities is severely restricted by geofence. The city-wide airspace availability reduces from $96.71 \%$ to $89.61 \%$ in San Francisco, and $89.36 \%$ to $66.76 \%$ in New York. From the correspondence analysis, regions with the HH category cannot be observed in both cities as shown in Fig. 7 (c) \& (d). However, in New York, cluster 2 and 5 are observed to change in category due to temporal change. Cluster 2 (Midtown) and 5 (Financial District) are classified as LL category at nighttime and HL category at daytime, respectively. In the HL category, airspace availability is low, but potential demand is high, hence balancing between them is crucial. Accordingly, efficient traffic flow management is needed in the Midtown and Financial District during the daytime. In addition, regions with low airspace availability need to consider urban canyon effect, which can have a significant impact on energy, wind speed, and direction of UAM flights [23]. Even though there does not exist $\mathrm{HH}$ category in both cities when considering geofence, Cluster 4 in both cities could be a strong candidate of $\mathrm{HH}$ category. Cluster 4 of San Francisco is in the Inner Sunset area. In case of New York, Cluster 4 is an area that includes East Village-Greenwich Village-Chelsea- Hell's Kitchen- Upper Westside.

In this study, we utilized 3D geodemographic datasets in the cities of San Francisco, and Manhattan, and examined the feasibility of UAM in those urban areas. To assess operational 
and economic feasibility, building footprint and population data with temporal changes were used. Regionalization was conducted to delineate regional boundaries by preserving spatial contiguity and grouping regions having similar characteristics by applying SKATER technique. Regionalization results captured the combined effect of airspace availability and population. Then, correspondence analysis was performed for the regions generated from the results of regionalization, and feasibility was assessed for each region.

Major contribution of our approach is assessing the feasibility of UAM in urban areas by incorporating airspace availability and potential demand. By providing region maps of each city that can readily identify regions of similar UAM operational and population characteristics with spatial continuity and feasibility, our approach can provide a useful basis for management for UAM applications in urban areas. In addition, it not only contributes to the process of managing and controlling the use of urban space but also to inferring the package delivery target area identification, and station placement for UAM application.

\section{Conclusion}

In this study, we utilized 3D geodemographic datasets in the cities of San Francisco, and Manhattan, and examined the feasibility of UAM in those urban areas. To assess operational and economic feasibility, building footprint and population data with temporal changes were used. Regionalization was conducted to delineate regional boundaries by preserving spatial contiguity and grouping regions having similar characteristics by applying SKATER technique. Regionalization results captured the combined effect of airspace availability and population. Then, correspondence analysis was performed for the regions generated from the results of regionalization, and feasibility was assessed for each region.

Geodemographic feasibility of each city was successfully demonstrated. Intuitively, major commercial areas in each city showed low airspace availability and high population concentration during the daytime. In San Francisco, regionalization results showed that the regional district located in the north east corner was classified as a HH category during the daytime. On the other hand, in New York, airspace restriction due to densely populated sky-scrappers becomes the main limiting factor in UAM application. After airspace restriction, regional boundaries were further subdivided, and strong candidates of $\mathrm{HH}$ category were found in Inner Sunset in San Francisco, and the area encompassing East VillageGreenwich Village-Chelsea- Hell's Kitchen- Upper Westside in Manhattan. Note that in correspondence analysis, the criteria for high and low population density and air availability can be reset from the user's perspective.

Major contribution of our approach is assessing the feasibility of UAM in urban areas by incorporating airspace availability and potential demand. By providing region maps of each city that can readily identify regions of similar UAM operational and population characteristics with spatial continuity and feasibility, our approach can provide a useful basis for management for UAM applications in urban areas. In addition, it not only contributes to the process of managing and controlling the use of urban space but also to inferring the package delivery target area identification, and station placement for UAM application.

\section{Acknowledgements}

This work was supported(researched) by the fourth Stage of Brain Korea FOUR 21 Project in 2021 and was also supported by the National Research Foundation of Korea (NRF) grant funded by the Korea government (MSIT) (No. 2020R1A2C2010200).

\section{References}

[1] Roca-Riu, M., Menendez, M., Dakic, I., Buehler, S., \& Ortigosa, J., 2020. Urban space consumption of cars and buses: an analytical approach. Transportmetrica B: Transport Dynamics, 8(1), 237-263.

[2] Shen, T., Hong, Y., Thompson, M. M., Liu, J., Huo, X., \& Wu, L., 2020. How does parking availability interplay with the land use and affect traffic congestion in urban areas? The case study of Xi'an, China Sustainable Cities and Society, 57, 102126.

[3] Thipphavong, D. P., Apaza, R., Barmore, B., Battiste, V., Burian, B., Dao, Q., ... \& Idris, H. R., 2018. Urban air mobility airspace integration concepts and considerations. In 2018 Aviation Technology, Integration, and Operations Conference (p. 3676).

[4] Baur, S., Schickram, S., Homulenko, A., Martinez, N., \& Dyskin, A., 2018. Urban air mobility: The rise of a new mode of transportation. Roland Berger Focus.

[5] Rothfeld, R., Straubinger, A., Fu, M., Al Haddad, C., \& Antoniou, C., 2020. Urban air mobility. In Demand for Emerging Transportation Systems (pp. 267-284). Elsevier.

[6] Goyal, R., 2018. Urban air mobility (UAM) market study, NASA Technical Report HQ-E-DAA-TN65181, NASA Headquarters, Washington, DC, available from: https://ntrs.nasa.gov/search.jsp?R=20190001472 [accessed 24 May 2020].

[7] Cho, J., \& Yoon, Y., 2018. How to assess the capacity of urban airspace: A topological approach using keep-in and keep-out geofence. Transportation Research Part C: Emerging Technologies, 92, 137-149.

[8] Cho, J., \& Yoon, Y., 2019. Extraction and Interpretation of Geometrical and Topological Properties of Urban Airspace for UAS Operations. In 13th USA/EUROPE Air Traffic Management R\&D Seminar.

[9] Vascik, P. D., Cho, J., Bulusu, V., \& Polishchuk, V. 2020. Geometric Approach Towards Airspace Assessment for Emerging Operations. Journal of Air Transportation, 1-10.

[10] Haggett, P., Cliff, A. D., \& Frey, A., 1977. Locational analysis in human geography (Vol. 2, pp. 259-605). London: Arnold.

[12] Guo, D., 2008. Regionalization with dynamically constrained agglomerative clustering and partitioning (REDCAP). International Journal of Geographical Information Science, 22(7), 801-823.

[13] Kugler, T. A., Manson, S. M., \& Donato, J. R. 2017. Spatiotemporal aggregation for temporally extensive international microdata. Computers, environment and urban systems, 63, 26-37.

[14] Assunção, R. M., Neves, M. C., Câmara, G., \& da Costa Freitas, C., 2006. Efficient regionalization techniques for socio-economic geographical units using minimum spanning trees. International Journal of Geographical Information Science, 20(7), 797-811.

[15] Esri., 2016 Methodology statement: 2016 Esri daytime population. Esri, Redlands

[16] City and County of San Francisco., 2020. Building Footprints. DataSF. Available from: https://data.sfgov.org/Geographic-Locationsand-Boundaries/Building-Footprints/ynuv-fyni [Accessed 1 Oct 2020] 
[17] City of New York., 2020. Building Footprints. NYC OpenData. Available from: https://data.cityofnewyork.us/HousingDevelopment/Building-Footprints/nqwf-w8eh [Accessed 1 Oct 2020]

[18] Ministry of Land, Infrastructure and Transportation of Japan., 2015. Aviation Act amended by Act No. 67. Available from: http://www.shugiin.go.jp/Internet/itdb_gian.nsf/html/gian/honbun/houa n/g18905075.htm [Accessed 2 Sep 2020].

[19] Transport Canada, 2016. Advisory Circular 600-004, Guidance Material for Operating Unmanned Air Vehicle Systems under an Exemption. Available from https://www.tc.gc.ca/eng/civilaviation/opssvs/ac-600-004-2136.html [Accessed 3 August 2020]

[20] Prim, R. C., 1957. Shortest connection networks and some generalizations. The Bell System Technical Journal, 36(6), 1389-1401.

[21] Sugar, C. A., \& James, G. M., 2003. Finding the number of clusters in a dataset: An information-theoretic approach. Journal of the American Statistical Association, 98(463), 750-763.

[22] Breunig, J., Forman, J., Sayed, S., Audenaerd, L., Branch, A., \& Hadjimichael, M., 2018. Modeling Risk-Based Approach for Small Unmanned Aircraft Systems.

[23] Vardoulakis, S., Fisher, B. E., Pericleous, K., \& Gonzalez-Flesca, N. 2003. Modelling air quality in street canyons: a review. Atmospheric environment, 37(2), 155-182. 